PROCEEDINGS OF THE

AMERICAN MATHEMATICAL SOCIETY

Volume 131, Number 8, Pages 2593-2596

S 0002-9939(02)06773-4

Article electronically published on November 14, 2002

\title{
COVERING $\mathbb{R}$ WITH TRANSLATES OF A COMPACT SET
}

\author{
UDAYAN B. DARJI AND TAMÁS KELETI
}

(Communicated by Alan Dow)

\begin{abstract}
Motivated by a question of Gruenhage, we investigate when $\mathbb{R}$ is the union of less than continuum many translates of a compact set $C \subseteq \mathbb{R}$. It will follow from one of our general results that if a compact set $C$ has packing dimension less than 1 , then $\mathbb{R}$ is not the union of less than continuum many translates of $C$.
\end{abstract}

\section{INTRODUCTION}

When is $\mathbb{R}$ the union of less than continuum many translates of a given compact subset of $\mathbb{R}$ ? Of course, if the compact set has non-empty interior, then $\mathbb{R}$ is easily seen to be the union of countably many translates of the compact set. On the other hand, if we assume the continuum hypothesis, then it follows from the Baire category theorem that there is no such nowhere dense compact set.

Gary Gruenhage observed that it is consistent with ZFC that given a compact set of positive Lebesgue measure, one can find less than continuum many translates of it whose union is $\mathbb{R}$. Hence, for nowhere dense compact sets of positive Lebesgue measure the question whether $\mathbb{R}$ can be written as less than continuum many translates of the given set is independent of ZFC.

Gruenhage also showed that $\mathbb{R}$ is not the union of less than continuum many translates of the standard "middle 1/3 Cantor set". Motivated by these results, he asked the following natural question:

Problem 1.1. Is there a compact set of Lebesgue measure zero and less than continuum many translates of it whose union is $\mathbb{R}$ ?

Of course, a positive answer to this problem would require some extra settheoretic assumption.

For the sake of notational convenience, let us call a compact set $C \subseteq \mathbb{R}$ thin if it is true in ZFC that $\mathbb{R}$ is not the union of less than continuum many translates of $C$. (We remark here that our definition of thin has nothing do with the notion of thin in harmonic analysis.) Hence, Gruenhage's question is whether every compact set of Lebesgue measure zero is thin.

Received by the editors January 24, 2002 and, in revised form, March 14, 2002.

1991 Mathematics Subject Classification. Primary 03E15; Secondary 28A78.

The first author thanks the Fulbright Foundation and the Department of Analysis of Eötvös Loránd University for their hospitality.

The second author was supported by OTKA grant F 029768. 
Daniel Mauldin also asked a similar question. Namely,

Problem 1.2. Is every compact set of Hausdorff dimension less than 1 thin?

In this note, we show that if we consider packing dimension instead of Hausdorff dimension, then the answer is affirmative.

Ronnie Levy asked whether it is true that $\mathbb{R}$ is not the union of less than continuum many similar copies of the standard middle $1 / 3$ Cantor set. We show that the answer is affirmative. We call a set similarity thin if it satisfies the definition of thin with the word "translates" replaced by "similar copies". We show that compact sets with packing dimension less than 1 are similarity thin. A more general result will be obtained, too. Finally, we shall see that Problem 1.1 would be independent of ZFC if we wanted a $G_{\delta}$-set instead of a compact set.

\section{Results}

For the sake of completeness, we first prove the following theorem due to Gruenhage. The proof given here is due to Márton Elekes.

Theorem 2.1 (Gruenhage). It is consistent with ZFC that given a compact set of positive measure, one can find less than continuum many translates of it whose union is $\mathbb{R}$.

Proof (M. Elekes). It is consistent with ZFC that there is a set $A \subseteq \mathbb{R}$ of cardinality less than the continuum which has positive Lebesgue outer measure. (See e.g. [1].) Let $C$ be a compact set of positive measure. By a variant of the well-known theorem of Steinhaus the sum of a measurable set with positive measure and a set with positive outer measure contains an interval. Hence, $A+C$ contains an interval. Now, let $T=\mathbb{Q}+A$. Then, $T$ is a set with cardinality less than that of the continuum and $T+C=\mathbb{R}$.

The basic idea behind our main result is the following simple fact. Recall that set $A \subseteq \mathbb{R}$ is similar to set $B$ if there are numbers $s, t, s \neq 0$ such that $B=t+s \cdot A$.

Lemma 2.2. Let $C$ be a compact set. If there is a perfect set $P$ such that $(t+s \cdot C) \cap P$ is countable for every $t$ and every $s \neq 0$, then $C$ is similarity thin.

If $C \subseteq \mathbb{R}$, then $C^{n}=\left\{\left(p_{1}, p_{2}, \ldots, p_{n}\right): p_{i} \in C\right.$ for all $\left.1 \leq i \leq n\right\}$. If $A \subseteq \mathbb{R}^{n}$, then $A_{*}=\left\{\left(x_{1}, x_{2}, \ldots, x_{n}\right) \in A:(i \neq j) \Longrightarrow\left(x_{i} \neq x_{j}\right)\right\}$. We define $F_{n}: \mathbb{R}^{n+2} \rightarrow$ $\mathbb{R}^{n}$ by $F_{n}\left(x_{1}, x_{2}, \ldots, x_{n}, s, t\right)=\left(t+s x_{1}, \ldots, t+s x_{n}\right)$.

Lemma 2.3. Suppose that $C$ and $P$ are compact sets. If a similar copy of $C$ intersects $P$ in at least $n$ points, then $F_{n}\left(C^{n} \times(\mathbb{R} \backslash\{0\}) \times \mathbb{R}\right) \cap P_{*}^{n} \neq \emptyset$.

Proof. Let $y_{1}, y_{2}, \ldots, y_{n}$ be $n$ distinct points of $P$ which are contained in some similar copy of $C$. Then $\left(y_{1}, \ldots, y_{n}\right) \in F_{n}\left(C^{n} \times(\mathbb{R} \backslash\{0\}) \times \mathbb{R}\right) \cap P_{*}^{n}$.

The following lemma is rather well-known. A far more general result can be found in [4. (Similar ideas were later used in [3].) We thank the referee for pointing this out.

Lemma 2.4. Fix a positive integer $n$. Suppose that $M \subseteq \mathbb{R}^{n}$ is an $F_{\sigma}$, first category set. Then, there is a perfect set $P \subseteq \mathbb{R}$ such that $P_{*}^{n} \cap M=\emptyset$. 
Let us recall some terminology and facts from basic geometric measure theory. If $A \subseteq \mathbb{R}^{n}$, then $\operatorname{dim}_{P}(A)$ and $\operatorname{dim}_{H}(A)$ denote the packing dimension and the Hausdorff dimension of $A$, respectively. (See the definitions and basic properties e.g. [2.)

Theorem 2.5. Every compact subset of $\mathbb{R}$ with packing dimension less than 1 is similarity thin. That is, less than continuum many similar copies of a compact set with packing dimension less than 1 cannot cover the real line.

Proof. Let $C \subseteq \mathbb{R}$ be a compact set with packing dimension less than 1 . By Lemma2.2, it will suffice to show that there is a perfect set $P$ such that $(t+s \cdot C) \cap P$ is finite for all real $t, s$ with $s \neq 0$.

Recall (see e.g. [2]) that for packing dimension, we have for Borel sets $A, B$,

$$
\operatorname{dim}_{H}(A \times B) \leq \operatorname{dim}_{P}(A \times B) \leq \operatorname{dim}_{P}(A)+\operatorname{dim}_{P}(B) .
$$

Hence, we may choose $n$ sufficiently large so that $\operatorname{dim}_{P}\left(C^{n}\right)<n-2$, which, in turn, implies that $\operatorname{dim}_{P}\left(C^{n} \times(\mathbb{R} \backslash\{0\}) \times \mathbb{R}\right)<n$ and hence

$$
\operatorname{dim}_{H}\left(C^{n} \times(\mathbb{R} \backslash\{0\}) \times \mathbb{R}\right)<n .
$$

We note that $F_{n}$ is countably Lipschitz, i.e. we can decompose the domain of $F_{n}$ into countably many compact sets $\left\{A_{i}\right\}$ so that $F_{n} \mid A_{i}$ is Lipschitz. Let $B_{i}=$ $\left(C^{n} \times(\mathbb{R} \backslash\{0\}) \times \mathbb{R}\right) \cap A_{i}$. Then, since Lipschitz maps clearly cannot increase Hausdorff dimension, we have that $F_{n}\left(B_{i}\right)$ is a compact set with $(n-2)$ dimensional Lebesgue measure zero and hence is of first category. However, $F_{n}\left(C^{n} \times(\mathbb{R} \backslash\{0\}) \times \mathbb{R}\right)=\bigcup_{i=1}^{\infty} F_{n}\left(B_{i}\right)$. Therefore, $F_{n}\left(C^{n} \times(\mathbb{R} \backslash\{0\}) \times \mathbb{R}\right)$ is an $F_{\sigma}$ first category set. By Lemma 2.4, we have that there is a perfect set $P \subseteq \mathbb{R}$ such that $P_{*}^{n} \cap F_{n}\left(C^{n} \times(\mathbb{R} \backslash\{0\}) \times \mathbb{R}\right)=\emptyset$. By Lemma 2.3. we have that no similar copy of $C$ intersects $P$ in more than $n$ points. By Lemma 2.2, we have that $C$ is similarity thin.

Theorem 2.5 can be easily generalized to any countably Lipschitz, finite (say, $k$ ) parameter images instead of similar copies. One can easily check that by replacing $(x, s, t) \rightarrow t+s x$ by any other countably Lipschitz function $f: \mathbb{R} \times H \rightarrow \mathbb{R}$, where $H \subseteq \mathbb{R}^{k}$ replaces $(\mathbb{R} \backslash\{0\}) \times \mathbb{R}$ as the set of possible parameters and replacing 2 by $k$ at some points, the same way as above, we can get the following generalization.

Theorem 2.6. Let $H \subseteq \mathbb{R}^{k}$ be a set of possible parameters, and let $f: \mathbb{R} \times H \rightarrow \mathbb{R}$ be a countably Lipschitz function. If $C$ is a compact set with packing dimension less than 1 , then less than continuum many sets of the form $f(C, h)(h \in H)$ cannot cover the real line.

\section{REMARKS}

As in Lemma 2.2, a negative answer to the original Problem 1.1 would follow from a negative answer to the following question:

Problem 3.1. Is there a compact set $C$ of Lebesgue measure zero such that every perfect set intersects at least one of the translates of $C$ in uncountably many points?

A positive answer to this question would only show that this method cannot solve Problem 1.1 But to this problem one can imagine (contrasted to Problem 1.1) a positive answer in ZFC. 
Both problems seem just as hard if "compact" is replaced by " $F_{\sigma}$ ". However, it is consistent with ZFC that there is a set of Lebesgue measure zero and less than continuum many translates of it whose union is $\mathbb{R}$ (see e.g. [1]). In fact, if there exists a set of second category with cardinality less than the continuum (which is consistent with ZFC, see e.g. [1]), then any residual set of Lebesgue measure zero has this property. (Since, as one can easily check, the sum of a set of second category and a residual set is $\mathbb{R}$.) Since there exist residual $G_{\delta}$ sets with Lebesgue measure zero, this means that if we replaced "compact" in Problems 1.1 and 3.1 by " $G_{\delta}$ ", then the positive answers would be consistent with ZFC.

\section{REFERENCES}

[1] T. Bartoszyński, H. Judah, Set theory: on the structure of the real line, A K Peters, 1995. MR 96k:03002

[2] P. Mattila, Geometry of Sets and measures in Euclidean Spaces, Cambridge University Press, 1995. MR 96h:28006

[3] R. D. Mauldin, On the Borel Subspaces of algebraic structures, Indiana University Math. J. 29 (1980), 261-265. MR 81i:54027

[4] J. Mycielski, Independent sets in topological algebras, Fund. Math. 55 (1964), 139-147. MR 30:3855

Department of Mathematics, University of Louisville, Louisville, Kentucky 40292

E-mail address: ubdarj01@athena.louisville.edu

Department of Analysis, EÖtvös Loránd University, PÁzmány Péter sétány 1/C, H1117 Budapest, Hungary

E-mail address: elek@cs.elte.hu 